\title{
Pseudoquiste peritoneal: Una complicación inusual en paciente con diálisis peritoneal
}

\author{
Alfredo Chama N. ${ }^{1}$, Cuauhtémoc Borja Villa ${ }^{1}$, Jorge Farell R. ${ }^{1}$ y Ana Paula Ruiz F. ${ }^{1}$
}

\section{Peritoneal pseudocysts: An unusual complication in a patient with peritoneal dialysis}

\begin{abstract}
Introduction: Renal replacement therapy with peritoneal dialysis is now a well-established, mature treatment modality for End-Stage Renal Disease. Patient outcomes with peritoneal dialysis are comparable than those with hemodialysis so does a more cost-effectiveness modality, especially in developing countries. Some complications of peritoneal dialysis are peritonitis, leaks, hernias, ultrafiltration failure, and catheter dislocation. One of the rare complications of peritoneal dialysis is peritoneal pseudocyst formation. Clinical Case: We report one such case of a patient with a history of renal replacement therapy managed on long-term peritoneal dialysis, which presents as a complication a peritoneal pseudocyst. Key words: complications; peritoneal dialysis; peritoneal pseudocysts.
\end{abstract}

\section{Resumen}

Introducción: La terapia de sustitución renal con diálisis peritoneal es una modalidad segura para la enfermedad renal crónica terminal. Los resultados son comparables con pacientes en hemodiálisis por lo que lo hace una modalidad costo efectiva, especialmente en países en vía de desarrollo. Algunas complicaciones que se pueden presentar debido a diálisis peritoneal son: peritonitis, fuga, hernias, falla de filtración y disfunción del catéter. Una de las complicaciones infrecuentes de la diálisis peritoneal es la formación de un pseudoquiste peritoneal. Caso Clínico: Presentamos un caso de un paciente en terapia de sustitución renal con diálisis peritoneal, el cual presenta un pseudoquiste peritoneal como complicación de diálisis peritoneal.

Palabras clave: complicaciones; diálisis peritoneal; pseudoquiste peritoneal.

\section{Introducción}

La terapia de sustitución renal (TSR) con diálisis peritoneal es una modalidad segura para la enfermedad renal crónica terminal (ERC). La prevalencia global de la ERC es cercana al 9\%, representando aproximadamente 700 millones de casos en el mundo, condiciona un deterioro en la calidad de vida, especialmente cuando se requiere TSR con diálisis peritoneal (DP) o hemodiálisis $(\mathrm{HD})^{1}$.

Un número considerable de pacientes se encuentran en DP en países en vía de desarrollo, debido a que en algunas instituciones se considera una alternativa, superior en comparación con la HD, especialmente por una modalidad ambulatoria, satisfacción del paciente y costo efectividad. La finalidad del tratamiento de DP es mejorar la calidad de vida y reducir la necesidad de tratamiento intrahospitalario $^{2}$. A pesar de los beneficios que ha demostrado la DP, no se encuentra exenta de complicaciones. Algunas de las complicaciones bien conocidas de la DP son la peritonitis, fuga, hernias, falla de filtración y disfunción del catéter. El pseudoquiste peritoneal (PP) es una complicación rara secundaria a DP, el cual contiene líquido y se encuentra aislado de los órganos abdominales, se han reportado pocos casos en la literatura ${ }^{3,4}$.

Presentamos el caso de un paciente masculino con antecedente de DP de 2 años de evolución, el cual desarrolló un PP que ameritó manejo quirúrgico. El objetivo es describir la presentación clínica, el abordaje diagnóstico y el manejo ofrecido al paciente.
Departamento de Cirugía General Hospital Central Sur de Alta Especialidad, Petróleos Mexicanos. Ciudad de México, México.

Recibido 2020-08-7 y aceptado 2020-10-8

Correspondencia a: Dr. Alfredo Chama N. alfredchamaa@gmail.com 


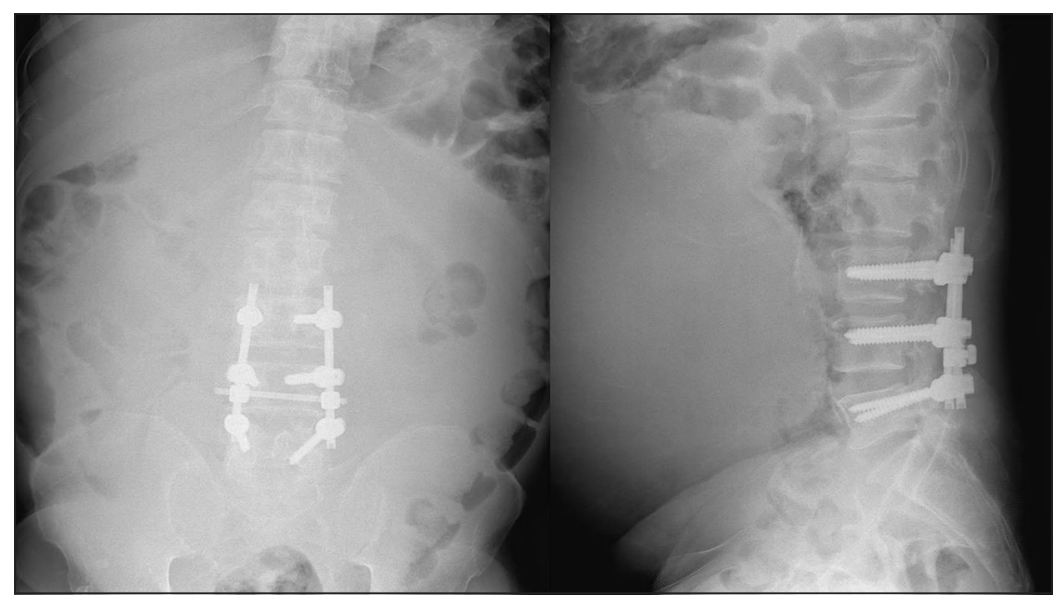

Figura 1. Radiografía anteroposterior y lateral con lesión ovoidea que abarca cavidad abdominal.

\section{Caso clínico}

Paciente hombre de 67 años con antecedente de hipertensión arterial y diabetes mellitus tipo 2 de 13 años de evolución. Diagnosticado con ERC de 4 años de evolución. Se encuentra en TSR desde hace dos años con DP. Durante el segundo año de DP se retira catéter por presentar episodios recurrentes de peritonitis refractarias al tratamiento médico.

El servicio de nefrología decidió TSR con HD. Dos meses posteriores al retiro del catéter de DP, inició con dispepsia, pérdida de peso y aumento de perímetro abdominal. $\mathrm{Al}$ interrogatorio refirió síntomas de plenitud gástrica, así como dolor abdominal tipo cólico en mesogastrio con irradiación a hipogastrio. Durante la exploración física, se observó un perímetro abdominal de $117 \mathrm{~cm}$, con presencia de un tumor adherido a planos profundos, no desplazable, sin dolor a la palpación superficial o profunda. No se encontraron signos de irritación peritoneal.

Se solicitó radiografía anteroposterior y lateral de abdomen, con evidencia de lesión ovoide que abarca meso e hipogastrio con desplazamiento de contenido abdominal (Figura 1). Se complementó abordaje diagnóstico con realización de tomografía abdominal donde se observó una lesión quística de $26 \times 11 \mathrm{~cm}$ de diámetro, con una densidad de -2UH y un volumen de $2.991 \mathrm{ml}$, compatible con un PP (Figura 2).

Inicialmente decidimos drenaje por paracentesis con evacuación de material citrino, con citoquímico compatible con líquido inflamatorio. Se repitió el procedimiento en 4 ocasiones, con recurrencia a las dos semanas de la evacuación. El drenaje promedio fue de 110 a 270 mililitros por evento. Debido a la recurrencia se ofrece intervención quirúrgica.

Se realizó laparotomía exploradora con hallazgos de un quiste simple de $25 \times 20 \mathrm{~cm}$ de diámetro, con múltiples adherencias al lóbulo hepático derecho y en ambas correderas parietocólicas (Figura 3). Se disecó la cápsula del peritoneo con escisión completa de lesión quística (Figuras 4 y 5).

El reporte histopatológico documenta una pieza con dimensiones de $27 \times 23 \times 10 \mathrm{~cm}, 2.000 \mathrm{ml}$ de contenido sanguinolento con un peso de $3.200 \mathrm{~g}$, compatible con pseudoquiste peritoneal. El paciente cursó su posoperatorio inmediato con adecuada evolución y es dado de alta a las $72 \mathrm{~h}$ del procedimiento. Continuó seguimiento por consulta externa de cirugía general, con alta a los 2 meses. A los dos años de seguimiento, el paciente continúa con TSR con HD. Sin presentar recidiva del pseudoquiste peritoneal. 


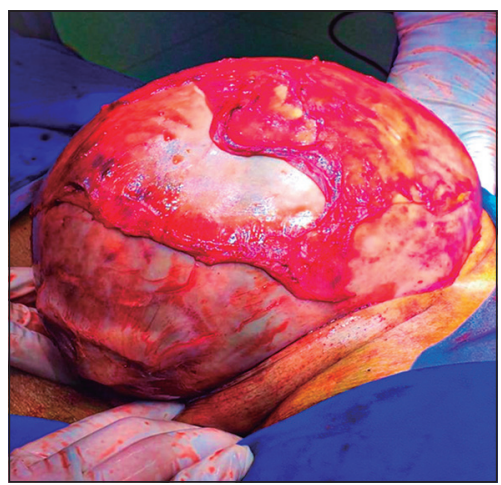

Figura 4. Liberación del pseudoquiste peritoneal

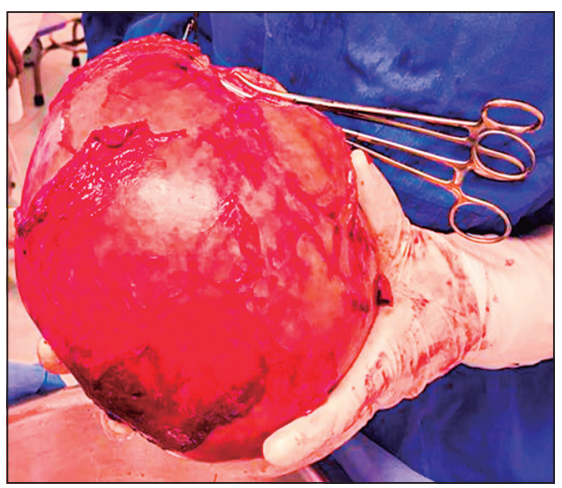

Figura 5. Pseudoquiste peritoneal resecado.

\section{Discusión}

La ERC es una enfermedad que progresa, por lo que genera un aumento en la necesidad TSR que sea costo-efectiva. En países desarrollados, los resultados de los pacientes con DP son comparables o mejores que aquellos con HD. A pesar de ser un método muy utilizado por presentar algunas ventajas (bajo costo y posibilidad de manejo ambulatorio), se asocia con complicaciones potencialmente graves. Una complicación poco frecuente que se ha descrito es la presencia del PP. Se han documentado hasta el momento 6 casos de PP $(1985,1991,2006$ y 3 casos en 2010) y 1 caso con recurrencia al tratamiento en $2018^{3,4}$.

El PP es una complicación infrecuente pero peligrosa de la DP asociada a un mayor riesgo de peritonitis recurrente ${ }^{3}$, tal como se muestra en nuestro caso. Generalmente los PP se encuentran como complicación de una derivación ventriculoperitoneal ${ }^{6}$. El reporte histológico de los PP generalmente muestra células de inflamación como en la serie de casos que se han reportado ${ }^{4}$. En nuestro paciente solo se observó proceso inflamatorio con contenido sanguinolento. En algunos casos se puede observar una membrana fibrosa inflamada, que se puede asociar como el resultado de una peritonitis aguda que conduce al desarrollo de la membrana fibrosa ${ }^{7}$.

El proceso de formación PP y el manejo integral no se encuentra estandarizado debido a los limitados casos que se han reportado. En los pacientes con DP se desconoce si es consecuencia de una irritación crónica del peritoneo o como resultado de una infección bacteriana recurrente. El tratamiento por mínima invasión por medio de intervencionismo guiada por ultrasonido o tomografía parece ser una opción viable para pacientes con alto riesgo para cirugía. Aunque, si el PP se presenta loculado, podría ser una limitante para el procedimiento, con riesgo de recidiva e infección. La resección quirúrgica de un PP sintomático suele ser curativa, como se realizó en nuestro paciente, es necesario realizar la resección de la cápsula o membrana del PP para evitar nuevamente su formación ${ }^{4}$.

\section{Conclusión}

La diálisis peritoneal continúa siendo una modalidad de tratamiento útil para pacientes con enfermedad renal crónica avanzada. Sin embargo, pueden presentarse complicaciones graves y recurrentes que suelen ser desafiantes de diagnosticar y tratar, tal como se muestra en nuestro caso un pseudoquiste peritoneal el cual se desconoce la causa y el mecanismo de desarrollo. Se requiere de más estudios para evaluar el manejo integral y los factores que contribuyen al desarrollo para integrar un tratamiento estandarizado.

\section{Responsabilidades éticas}

Protección de personas y animales. Los autores declaran que para esta investigación no se han realizado experimentos en seres humanos ni en animales.

Confidencialidad de los datos. Los autores declaran que en este artículo no aparecen datos de pacientes.

Conflictos de interés: no hay. 


\section{CASOS CLÍNICOS}

\section{Bibliografía}

1. Cockwell P, Fisher LA. The global burden of chronic kidney disease. Lancet. 2020;395(10225):662-4. doi: 10.1016/ S0140-6736(19)32977-0.

2. Brown EA, Bargman J, Van Biesen W, Chang MY, Finkelstein FO, Hurst $\mathrm{H}$, et al. Length of time on peritoneal dialysis and encapsulating peritoneal sclerosis - Position paper for ISPD: 2017 update. Perit Dial Int. 2017;37:362-74. doi: 10.3747/pdi.2017.00018.

3. Nagaraj S, Khan MH, Afroza F.
Recurrent Peritoneal Pseudocyst: A Rare Complication of Peritoneal Dialysis. Cureus 2018;10:e3043. doi: 10.7759/ cureus.3043.

4. Baer G, Wagner A, Selbach J, Otto M, Weiner SM. Abdominal Pseudocysts Following Peritoneal Dialysis-Associated Peritonitis: A Report of 3 Cases. Am J Kidney Dis. 2010;55:e15-e91. doi: 10.1053/j. ajkd.2009.12.019.

5. Li PK, Chow KM, Van de Luijtgaarden MW, Johnson DW, Jager KJ, Mehrotra $\mathrm{R}$, et al. Changes in the worldwide epidemiology of peritoneal dialysis. Nat Rev Nephrol. 2017;13:90-103. doi: 10.1038/nrneph.2016.181.

6. Rainov N, Schobess A, Heidecke V, Burkert W. Abdominal CSF pseudocysts in patients with ventriculo- peritoneal shunts. Report of fourteen cases and review of the literature. Acta Neurochir. (Wien) 1994;127:73-8.

7. Demirel AH, Ongoren AU, Karaglo N. Intraabdominal pseudocyst formation associated with continuous ambulatory peritoneal dialysis: a case report. Pakistan J Surg. 2006;22:183-4. 